\title{
CHARM: A Catalog of High Angular Resolution Measurements ${ }^{\star}$
}

\author{
A. Richichi ${ }^{1}$ and I. Percheron ${ }^{2}$ \\ 1 European Southern Observatory, Karl-Schwarzschildstr. 2, 85748 Garching bei München, Germany \\ 2 NEVEC, Leiden Observatory, University of Leiden, PO Box 9513, 2300 RA Leiden, The Netherlands
}

Received 9 November 2001 / Accepted 5 February 2002

\begin{abstract}
The Catalog of High Angular Resolution Measurements (CHARM) includes most of the measurements obtained by the techniques of lunar occultations and long-baseline interferometry at visual and infrared wavelengths, which have appeared in the literature or have otherwise been made public until mid-2001. A total of 2432 measurements of 1625 sources are included, along with extensive auxiliary information. In particular, visual and infrared photometry is included for almost all the sources. This has been partly extracted from currently available catalogs, and partly obtained specifically for CHARM. The main aim is to provide a compilation of sources which could be used as calibrators or for science verification purposes by the new generation of large ground-based facilities such as the ESO Very Large Interferometer and the Keck Interferometer.
\end{abstract}

Key words. techniques: high angular resolution - techniques: interferometric - catalogs - occultations stars: binaries: general - stars: fundamental parameters

\section{Introduction}

The need to obtain observations at high angular resolution has increased constantly in the recent decades, stimulating the invention of specialized, and often technically complicated, astronomical techniques. On one side, methods such as speckle interferometry, aperture masking and adaptive optics, aimed at overcoming the limits set by atmospheric turbulence, have been developed for use with a single aperture. On the other side, long-baseline interferometry, with various schemes of fringe detection, has been employed to combine two or more telescopes and to achieve the diffraction limit of an equivalent telescope as large as their separation. Finally, lunar occultations have been used to obtain high angular resolution information on stars covered by the apparent motion of the Moon, using the lunar limb as a diffracting straight edge.

Of all the various techniques for high angular resolution, long-baseline interferometry is one of those which is foreseen to be used more intensively in the near future. The demonstration of its capabilities by several facilities in the last couple of decades has spawned the design and construction of a number of modern, large-scale interferometers, which are expected to dominate the domain of

\footnotetext{
Send offprint requests to: A. Richichi,

e-mail: arichich@eso.org

* The Catalog is available in electronic form at the CDS via anonymous ftp to cdsarc.u-strasbg.fr (130.79.128.5) or via

http://cdsweb.u-strasbg.fr/cgi-bin/qcat?J/A+A/386/492, and from the authors on CD-Rom.
}

high angular resolution observations at the high end in the coming years. Two examples of such facilities are the ESO Very Large Telescope Interferometer (VLTI, Glindemann et al. 2000) and the Keck Interferometer (KI, Colavita \& Wizinowich 2000), both of which employ a number of different telescopes of various sizes up to 8-10 $\mathrm{m}$, and several baselines up to about $200 \mathrm{~m}$. Both facilities have achieved first fringes in early 2001.

The use of such sensitive apertures with such long baselines will explore a range of angular resolutions and limiting magnitudes that has never been accessible before in combination. At the same time, this calls for the utmost accuracy in the calibration of the response of such interferometers. This task will require the use of stars with known angular diameters (or binary stars with known orbits), and for this it will be necessary to use on one side previously measured objects, and on the other side stars for which the angular diameter can be reliably estimated on the basis of current astrophysical models.

For these reasons, we believe that a compilation of all measurements at high angular resolution published so far is required. At the same time, such a compilation would provide a list of sources worthy of further investigations (such as, for example, pulsating stars), and that could serve as ideal targets for large modern interferometers.

We note that among other existing compilative works (see Sect. 2), only a fraction of the material which is suited to the specific range of angular resolution and sensitivity of modern interferometers is included. Also, no compilation has ever been made to list all measurements obtained by 
one technique, regardless of the type of results obtained. We stress that, for the purpose of finding suitable interferometric calibrators, negative results also can be useful, provided that an upper limit on the size of the unresolved source is present or can be inferred.

Given the wide range of resolution and sensitivity encompassed by the various techniques for high angular resolution, such a work must necessarily be split. Considering the needs of the new large facilities mentioned above, we have concentrated ourselves on results at the high-end of current technical possibilities, especially for what concerns angular resolution. In this paper, we present a compilation of all results published by the methods of lunar occultations and long-baseline interferometry, including whenever possible also results on unresolved sources.

We have restricted ourselves to the visual and near/thermal infrared, namely to wavelengths between $\approx 0.4$ and $\approx 20 \mu \mathrm{m}$. We have decided to include only values obtained through actual measurements in our compilation, in order to be homogeneous. Estimates obtained through indirect methods are of course also very valuable in this line of work, and are briefly described in Sect. 2 together with an overview of other existing catalogs of high angular resolution measurements. In Sect. 3 we provide some background on the techniques of lunar occultations and long-baseline interferometry, and in particular we comment on their specific characteristics of detection and sensitivity. We also provide a discussion of the importance of calibration measurements for such techniques. In Sect. 5 we describe the criteria used for our compilation, the organization and availability of the material, and its completeness. Finally, in Sect. 6 we consider some of the conclusions that can be drawn, in a statistical sense, from this compilation.

\section{Comparison with other catalogs}

Naturally, the use of these different techniques, each with its specific range of application, has produced a wealth of results which are difficult to organize in a homogenous database. This situation is reflected in the rather varied nature of the available literature.

White \& Feierman (1987, WF hereafter) compiled a list of angular stellar diameters measured by lunar occultations. Both for the completeness of the information and the discussion of the material, this work has proven quite useful as a reference source. However, the field of lunar occultation measurements has expanded considerably in the meantime, for what concerns the number of stars measured but in particular with respect to the accuracy of the measurements. In this sense, an updated compilation is desirable.

Fracassini et al. (1981) initiated a compilation of stellar radii, published in the literature and obtained by a large number of different methods. This resulted in the CADARS catalog, which after several revisions has recently been updated and considerably expanded by Pasinetti Fracassini et al. (2001). This compilation is quite extensive, including over 13000 radius determinations for almost 8000 stars. While this is a massive source of information, it must be noted that it includes a substantial number of very diverse methods, many of which are indirect. As a result, also the range of angular diameters, as well as of magnitudes of the sources, is quite broad.

Both the above compilations are restricted to angular diameters. For what concerns binary stars, the most important source of information, including high angular resolution measurements, is probably the WEB-based library kept by the CHARA group (Hartkopf et al. 2001). In particular, a catalog of interferometric measurements is provided, which however includes for the largest part measurements by methods which are restricted to the diffraction limit of a single telescope (notably, speckle interferometry), thereby including also sources with relatively large separations.

Another important source of information in the field of binary stars is the DSFILE list maintained by the International Occultation Timing Association (IOTA). It is based on a list of LO results on binary stars originally created by D. Dunham, and further expanded by T. Murray, M. Soma and H. Bulder to include results from other techniques as well (see Sect. 5.2).

Finally, it is worthy mentioning that recently a number of indirect methods to measure angular diameters have also flourished. These are generally based on the comparison of accurate photometry over a broad wavelength range, both in broad-band and narrow filters, with the emergent stellar flux predicted by model stellar atmosphere theory. Recent examples can be found in Blackwell \& Lynas-Gray (1994), and Cohen et al. (1999). In particular this latter work, which lists angular diameters for over 400 stars with rather good accuracy, is often used as a source of calibrators in interferometric work.

\section{The observational techniques}

As explained in the introduction, we have chosen to restrict the present compilation to the results obtained by two techniques, namely lunar occultations (LO) and longbaseline interferometry (LBI). These two techniques cover a range of resolutions which extends to $\$ 1$ milliarcsecond (mas). This is equivalent to the diffraction limit of a $200 \mathrm{~m}$ baseline at $1 \mu \mathrm{m}$. Therefore, this choice provides a database which is well suited to be used by modern large interferometers such as VLTI and KI, both for calibration and science verification purposes. It also provides a compilation of sources which deserve follow-up studies with high angular resolution techniques.

Given that each technique has its own technical challenges and its specific data reduction methods, the results are better understood if they are put in the context of the technique used. For this, we consider it useful to provide in this section a brief overview of the LO and LBI techniques. 


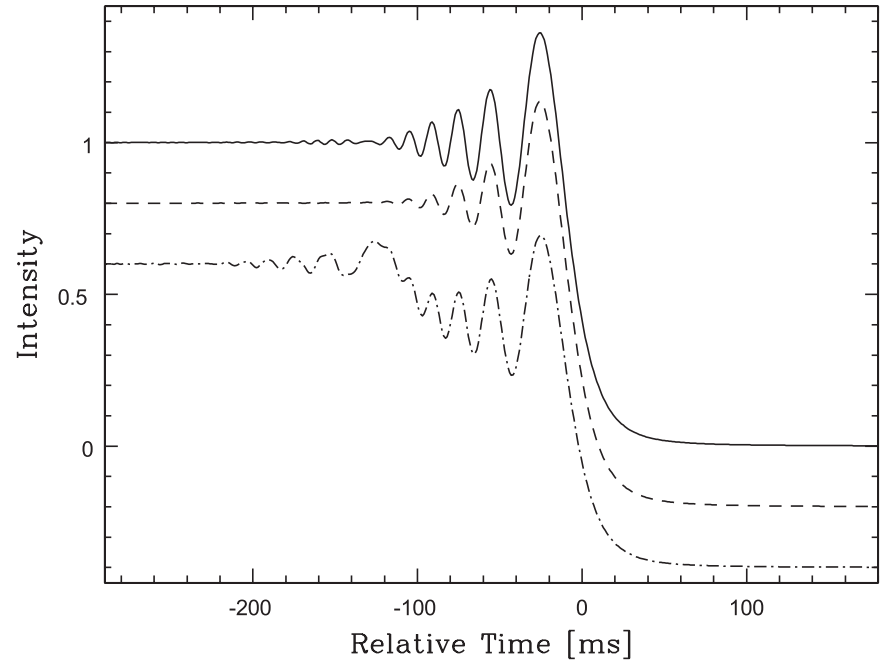

Fig. 1. Simulation of lunar occultation light curves under typical parameters for a $K$-band observation. The solid and dashed lines are obtained for stellar discs of 2 and 5 mas respectively. The dash-dotted line is for two unresolved stars separated by $0 . ' 2$ and with a 1:4 brightness ratio. The quantitative details of the observed diffraction patterns depend on the filter bandwidth, integration time, and telescope diameter. In these simulations, the background has been set to zero, the intensities before occultation have been normalized to unity, and arbitrary offset have been added. The time is counted from the time of geometrical occultation.

\subsection{Lunar occultations}

The technique of lunar occultations is based on the observation of the diffraction pattern produced by the lunar limb, when it covers a distant background source. The limb can be rather well approximated as an infinite straight edge, and this optical problem has a simple analytical solution in the case of a point source in monochromatic light. The diffraction pattern has a characteristic appearance, with fringes on one side following a precise intensity law, and a decreasing light distribution on the other side. The cases of sources with a finite size and of non-monochromatic light can be studied by numerical integrations from this standard pattern, as well as those of binary and multiple stars. As the lunar limb moves across the sky, the diffraction pattern moves across the telescope, producing typical light curves, some examples of which are also shown in Fig. 1.

High angular resolution information on the occulted source can be derived by studying such light curves. In particular, stellar angular diameters are relatively straightforward to determine, by fitting the amplitude of the fringes in the light curve. A least-squares fitting procedure is usually adopted to analyze the data, following a method initially described by Nather \& McCants (1970). Stars with a smaller angular diameter will produce fringes with a higher contrast, while for increasingly larger angular diameters the fringes tend to have less contrast, and eventually a smooth transition to the case of classical geometrical optics (i.e., no fringes) is observed. Depending on the wavelength, this occurs for angular diameters $\gtrsim 10$ 40 mas, above which the method is relatively inaccurate. At the other end, the method is limited in practice by factors such as atmospheric scintillation and instrumental effects, to angular sizes $\approx 1$ mas.

In addition to angular diameters, another ideal target for the method is that of binary (or multiple) stars, which give origin to light curves in which two (or more) independent diffraction patterns are observed, separated by time differences which are proportional to the separation of the components (see Fig. 1). A more difficult case is that of extended sources, for which no model is available. In this case, model-independent methods have been derived (see for instance Richichi 1989), but the solution is not unique.

LO constitute a very simple technique to attain what has been, for a very long time, the highest angular resolution possible at visual and near-IR wavelengths. Also the sensitivity limit is favorably comparable with that of other techniques, reaching about $K \approx 12$ on a 3.5 m-class telescope. It is also relatively independent from seeing conditions. Of course, the method can be used only on sources occulted by the Moon (about 10\% of the sky) and suffers from great limitations in repeatability.

Thanks to the novel possibility of obtaining what was then considered extremely high angular resolutions also with rather small telescopes, the LO method was widely used especially in the 1970's by many observers. However, the process of data analysis at that time was not always fully understood, and this resulted occasionally in optimistic error estimates. In the 1980's the method reached a more mature state, and it was also extended to the nearIR domain, where many of the sources of bias in the results are considerably decreased. In the last two decades, the method has been used by a more limited number of observers, but at the same time more rigorous data analysis methods have been employed (see for example Richichi et al. 1992) and the results have shown a much improved consistency and reliability.

\subsection{Long-baseline interferometry}

Stellar interferometry uses interference effects to improve the angular resolution of stellar observations. The coherent combination of two telescopes (more exactly, two independent sub-apertures of the same telescope) was demonstrated for the first time by Michelson \& Pease (1921), who provided the first direct measurement of the angular diameter of a star other than the Sun. However, the technique proved to be too difficult to be used routinely, and it remained essentially forgotten until Hanbury-Brown et al. (1974) invented an important variation, which however was applicable to a limited number of sources. Modern interferometry saw a second birth after the new observations of Labeyrie (1975). Since then, this technique has been developed by many teams to the level of a powerful and reliable method, with a consistent output of scientific results. 

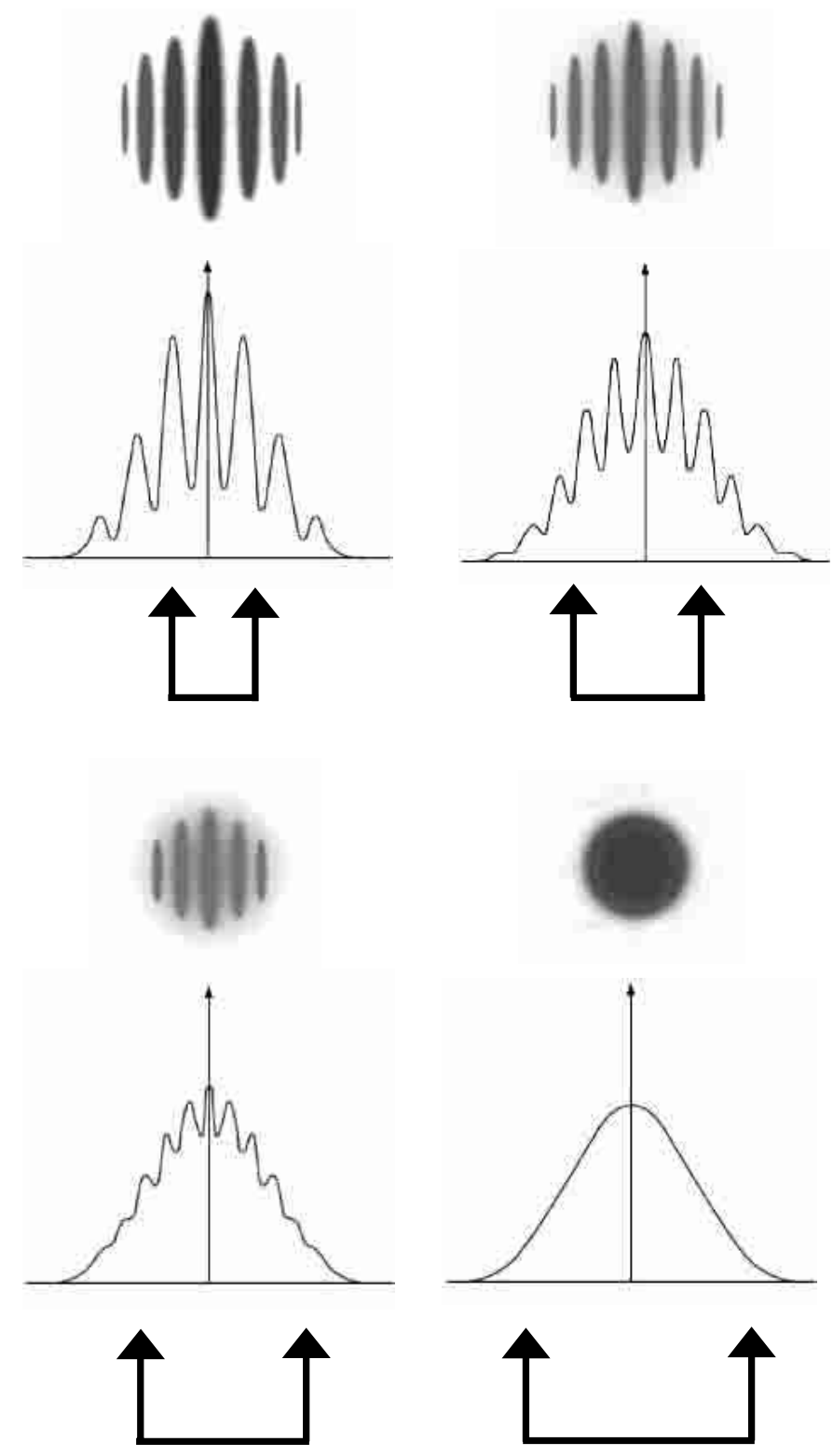

Fig. 2. Interference fringes on a stellar object are shown at different baselines. When the baseline between the two telescopes increases, the contrast of the fringes decreases. At a given baseline the fringes disappear. The diameter (or spatial parameters) of the object can be extracted from these data.

An updated summary of existing and planned interferometers has been given by Ridgway \& Roddier (2000).

An illustration of the basic principle of interferometry is provided in Fig. 2. There are perhaps two principal limitations to interferometric results: the distortion of the wavefronts due mainly to the atmospheric turbulence, and the limited number of baselines which does not allow a full coverage of the Fourier domain and therefore precludes actual imaging. For the first aspect, interferometers have been traditionally limited to small apertures, and therefore to relatively bright objects. However, recent technological advances, and in particular the availability of adaptive optics, are permitting the use of large mirrors. For the second aspect, Earth rotation, as well as the use of various baselines and to some extent of various wavelengths, can be used to fill the Fourier plane, and post-processing methods can be applied in suitable cases to produce "interferometric images". Another limitation in the accuracy of the measurements in LBI is the uncertainty in the determination of the instrumental transfer function (see Sect. 3.3).

Most of the results which have been obtained until now used only the amplitude of the complex visibility, and therefore could only answer a restricted range of scientific problems: examples are the determination of stellar diameters, the characteristic size and orientation of dust shells, the study of binary or multiple stars. A step forward in this sense will be permitted by the use of phase closure techniques, borrowed from the experience of radio interferometers (Baldwin et al. 1996). For this, it is necessary to combine three or more telescopes simultaneously, or to use the phase information from a nearby reference source (Delplancke et al. 2000).

\subsection{Calibrators}

In any process of scientific measurement, calibration of the measuring apparatus is a fundamental step. The situation is conceptually the same with the LO and LBI methods. To fully appreciate the quality of the published measurements and in particular of the associated error, the reader should be familiar with the process of calibration in the specific case of these two techniques. It is not seldom the case, that differences in the results obtained for the same source by different authors, can be largely explained by their different approach to this problem.

In the case of LO, the technique enjoys the unique situation that the diffraction phenomenon, which is the key to its power of angular resolution, takes place not at the telescope, but at the limb of the Moon. The major disturbances are caused by the instrumentation. For example, the finite size of the primary mirror produces a smearing of the diffraction fringes, especially when large telescopes are used: in the near-IR, a typical separation on the ground between the first two fringes is of about $20-50 \mathrm{~m}$, and this is reduced to about one-half in the visual range. Similar effects are caused by the finite integration time (during which the fringe system moves over the telescope), and by the finite bandpass of the wavelength filter (since diffraction is a chromatic phenomenon).

All these effects produce a bias towards angular diameters which appear larger than they are in reality. However, they are due to instrumental aspects which are (or should be) well measurable. Ridgway (1977) and more recently Richichi (1992), for example, have discussed the magnitude of such effects and ways to include them in the data reduction process. It must however be recognized that, especially in the first years of application of the LO technique, these facts were not known, or not largely applied. As a result, a large number of measurements are probably 
optimistic in their quoted errors, or in their results regarding resolved sources of very small angular diameter. We also note that the situation has considerably improved in more recent times, as mentioned in Sect. 3.1.

The problem of calibration for LBI is more difficult to solve, since in this case the instrumental response is determined, in addition to the technical specifications of the facility, also by the random effect of atmospheric turbulence. This latter introduces distortions on the wavefronts, which are different for each aperture of the interferometer. The problem can be solved only in a statistical way, assuming that the average properties of the atmospheric turbulence are the same on all apertures, and constant in time. Under these conditions, the calibration can be performed by measuring in close sequence the target source and a known calibration source, and ideally by interpolating the transfer function from various calibrators. The situation is even more stringent in the special case of phase-referenced interferometry, in which also the object phase is measured relative to a nearby reference star. Then, this latter must be in the same isoplanatic and isopistonic patch as the scientific object. The isopistonic angle is defined as the off-axis distance at which the visibility is reduced by $20 \%$ because of the differential piston introduced by the atmosphere (Esposito et al. 2000). In the near-IR, the limit is in practice $20-30^{\prime \prime}$, which significantly restricts the number of possible reference objects. The situation is even more critical at visual wavelengths, where the permitted angles are even smaller, and for some classes of sources such as extragalactic objects, which are typically in areas of the sky with a low density of field stars.

The astronomical calibrators necessary to measure the transfer function of interferometric arrays need to be unresolved at the given configuration and wavelength, or to have a very well known angular diameter. To provide an example, an interferometer working with a $100 \mathrm{~m}$ baseline at $\lambda=2.2 \mu \mathrm{m}$, and capable of achieving an intrinsic absolute accuracy of $0.1 \%$ on the fringe visibility, will need calibrators with an angular diameter that can be safely assumed to be $\lesssim 0.1$ mas. Alternatively, if for example one wishes to use a calibrator with a known angular diameter of 1 mas, this latter must be known to about 1:100. Additionally, one should consider whether the quoted angular diameter has been derived with or without the appropriate limb-darkening assumptions, and apply the necessary adjustments, for example for a different wavelength.

This illustrates the crucial problem of calibration for LBI, especially with high-accuracy, large modern interferometers. To solve it, it will be necessary to establish a database of calibrators, starting with well known objects at shorter baselines and moving on to the longer ones in a boot-strapping strategy. This has been one of the main motivations for the present work.

\section{Photometry}

Photometry is a fundamental part of the set of informations required to establish a database of sources for calibration and science verification of large interferometers. Obviously, it is necessary to know the brightness of the objects at the wavelengths of observation, in order to establish quantities such as the integration time and the expected signal-to-noise ratio (quantities that must be computed taking into account also the expected visibility).

However, photometry is required also for other important reasons. For example, it might be necessary to assess whether variability is present: this characteristic is usually considered a key parameter to reject possibly unsuitable calibrators. In the case of more complex objects, such as for example evolved stars, photometry at several wavelengths is the key to determine the spectral energy distribution of a source, which in turn can help to decide whether a source might have a very different appearance at different wavelengths. It is not seldom the case, that a source which is suitable to be used as a calibrator in a range of wavelengths, proves to be inadequate in another range.

We have made an effort to include in CHARM a comprehensive set of photometric measurements. These are collected from various sources, as described below. The emphasis has been on infrared wavelengths.

For each entry, we have identified a valid Simbad name (this is also included in the full version of CHARM), and we have extracted the $B$ and $V$ magnitudes from Simbad. For what concerns infrared wavelengths, we have queried, by means of the programs available at CDS, a number of catalogs including the Two Micron Sky Survey (TMSS, Neugebauer et al. 1969), the Catalog of Infrared Observations (CIO, Gezari et al. 1999), the Midcourse Space Experiment (MSX, Egan et al. 1999), the Two Micron All Sky Survey (2MASS, Skrutskie et al. 1997).

Additionally, CHARM includes a large number of novel photometric measurements, which have been obtained either specifically for this work, or have been borrowed from the private database of one of us (AR). This was particularly necessary, since many of the sources in CHARM are not covered by ordinary photometric cata$\operatorname{logs}$, as they fall in the gap of infrared magnitudes covered at one end by faint catalogs such as 2MASS, and at the bright end by TMSS.

These photometric measurements were obtained, mainly for LO sources, at facilities such as the TIRGO italian observatory, the ESO $1 \mathrm{~m}$ telescopes in La Silla, various telescopes from the Calar Alto observatory, and other. References to these photometric measurements and the instruments used can be found in Richichi et al. (1999, 2002). In a number of cases, in the absence of an actual measurement, the magnitude has been roughly estimated from the level of the signal during the LO event.

Furthermore, in the course of 2001 a number of measurements have been obtained with the DENIS instrument at the ESO $1 \mathrm{~m}$ telescope in La Silla. This has been part of a collaboration with the DENIS team, to obtain pointed observations of sources normally saturated in the DENIS 
and 2MASS surveys, and is described in a dedicated paper by Kimeswenger et al. (2002).

All the photometric data mentioned above are provided in the form of ASCII data files, which are accessed in the full version of CHARM by means of hyperlinks from the main table. The format of these photometric data is organized as one row for each measurement, reporting the wavelength or photometric band, the magnitude or flux with the associated error, and a short acronym to identify the source of the measurement and the date. This format is not rigidly specified, and at present it is not suitable to be automatically interpreted. Nevertheless, we have developed some limited programs in order to extract the $K$-band magnitudes which are published in the ASCII version of CHARM. When two or more measurements were available, we have retained in the CHARM table (see Sect. 5.3) the maximum magnitude.

\section{The database}

The collection of entries on high angular resolution measurements, which we refer to in a loose sense in this paper as the database, was originally created having in mind the needs for calibration and science verification of the ESO VLTI. For this reason, a database format had to be selected, which was easily accessible to all potential users, and that could support a number of features needed for the preparation and execution of observations.

The format chosen was that of an Excel spreadsheet, consisting of a main table and several secondary worksheets. Some of the supported features in the full version include: links to observational data, obtained by the VLTI, when available; links to the full Simbad documentation, which is regularly updated on disk (alternatively, hyperlinks to the actual Simbad server are available); links to images, in $10^{\prime} \times 10^{\prime}$ format, around each source, extracted from the Digital Sky Survey; links to photometric and spectroscopic data for each source, obtained in connection with the present work; links to the original bibliographical entry, which in turn is linked to a scanned version of the paper.

The Excel platform allows the user to sort and filter the entries, thereby permitting an easy selection according to user-defined constraints. It also allows embedded computations (which publish in one column the result of a formula based on entries in other columns), and it includes other features to keep track of statistics and to plot useful quantities.

Through specific worksheets, it is possible to update the database when needed, and to output other formats, such as ASCII, to other files. For example, one such worksheet is used when it is necessary to update all Simbad entries. Another worksheet is used to extract a catalog, which can then be converted into an ESO standard cata$\log$ and be used to point the interferometer.

For the purpose of the present paper, we restrict ourselves to some of the main entries only, and we provide a catalog which is organized as described in Sect. 5.3.
The full CD version, with a directory structure which enables the links to work properly, is self-contained on a CD-Rom. The CD-Rom is available from the authors upon request. The full Excel version has been tested under Windows 9x/NT only.

\subsection{Selection criteria}

In order to select valid entries for our compilation, we searched the literature using the ADS database. Given that this latter covers not only all main international journals, but a number of national publications and conference proceedings as well, our search could be considered already relatively satisfactory. In an effort to improve it, we asked several colleagues working in the field to indicate to us papers which might have not been included in the search described above. This resulted in the inclusion of a few additional papers, some of which in non-English languages, as well as of some results which were available from WEB sites or which covered results presented at conferences and not subsequently published. In most cases, such papers were available only in hardcopy, sometimes of less than optimal quality, and our scanned electronic copies reflect this.

Most of the material in the database, and presented in the catalog described in Sect. 5.3, has been assembled automatically. For this, we had to identify in each case a valid Simbad name. Subsequently, programs were written to query and extract from the Simbad database data such as coordinates with proper motions, cross-identifications, magnitudes, spectral types, radial velocities, parallaxes, variability. Similarly, the Digital Sky Survey maps have been extracted by interfacing to existing software available at ESO. For this part of the compilation, we rely on the accuracy of the above mentioned sources. One advantage of this approach is that, in principle, this process can now be replicated almost automatically for the whole source list. This will be useful to provide in the future regular releases with updated information.

\subsection{Completeness}

We stopped our search on July 1, 2001. Due to possible delays in the transmission of papers from the original sources to the ADS database, our compilation can be considered complete at least throughout the year 2000 .

We have explicitly searched the literature for results by LO starting from the year 1986, while prior to that date we have heavily relied on two sources of information, namely the WF and DSFILE catalogs mentioned in Sect. 2. The WF catalog can be considered complete until 1986, and has a detailed list of references and explanations. The DSFILE catalog is not published, but can be freely downloaded along with explanatory supplements and some auxiliary programs (H. Bulder, private communication). Given the nature and scope of this catalog, it cannot be considered complete, but it represented the best summary available to us of results published both in refereed journals and in more informal newsletters related to 
LO work on binary stars. We sorted and filtered the contents of DSFILE, to exclude stars observed with methods other than LO, and to include only those classified as certain or confirmed (thereby excluding doubtful events).

The use of WF and DSFILE in CHARM has the drawback that in many cases we have referenced these catalogs rather than the original papers. More importantly, since these catalogs contain only positive results, for papers published before 1986 we lack a reference to a large number of LO observations of unresolved sources. In particular, we note that the group led by D. Evans produced a long series of publications on photoelectric LO observations (Evans et al. 1986 and references therein), resulting in a database of thousands of stars observed. Regrettably, this material is not available in electronic format, we decided to include only those positive results which are referenced in DSFILE. However, we note that for many of the photoelectric observations published in the 1970's and 1980 's, the quality of the data is such that negative results have a limited application when compared to the calibration needs of modern interferometric facilities.

Regarding LBI results, we have tried to include all available literature, starting our search from the pioneering paper by Michelson \& Pease (1921).

\subsection{Catalog format}

The catalog includes 2432 entries, each representing a single measurement. In many cases several independent measurements are available for each source, and in some cases different names are used for the same source, as explained below. In total, 1625 unique sources are included. A list of cross-identifications and associations with table entries is provided in a separate file.

The data are presented as a tab-separated ASCII file, in which data are organized in columns. A total of 42 columns are logged. A sample of the catalog is illustrated in Table 1.

Columns 1 and 2 provide a numbering system. The first number is sequential, while the second one refers to the sequence of the entry in our original files. This is necessary for the full version, where entries can be sorted according to different criteria. For the version here described and provided as an ASCII catalog, the sources have been sorted according to their right ascension.

Column 3 is a mnemonic name. Column 4 is a code that describes the class of the source. The existing codes are listed in Table 2.

Columns 5 through 8 list some cross-references. These are extracted automatically from the Simbad database. The fields which are preferably extracted, when available, are the SAO, IRC, HD, AG, IRAS catalogs, followed by variable name, star name, and HR number. Additional fields are extracted, when the previous fields are not present to fill the four columns, in the order provided by Simbad.
Column 9 reports a code to identify the method of observation, either LO or LBI. Column 10 reports the wavelength of observation, if given in the paper. This is listed in different ways, depending on the type of measurement: one or two numbers, in which case it means $\lambda$ (and $\Delta \lambda$ ) in $\mu$ m; or a filter type, in which case the usual standard photometric names apply (for instance: $V, K, N$ ); or a symbol, for special or narrow filters. Examples can be: CVF (Circular Variable Filter), $\mathrm{H} \alpha, \mathrm{Br} \gamma$. Measurements obtained at several wavelengths simultaneously are labelled "mult".

Columns 11 and 12 report the angular diameter result and its error in the case of a uniform disc model, if reported in the original reference. These, as well as the next Cols. through 17, are expressed in mas. Column 13 reports an upper limit on the diameter of the source, when available. Columns 14 and 15 are the same as 11 and 12, for the case of a limb-darkened diameter, if reported in the original reference.

Column 16 is used for sources with an extended structure, for example those surrounded by a circumstellar dust shell. A "Y" indicates the existence of an extended structure, a listed number gives a characteristic size of the structure. Given that the geometry of the structure can be complex, as well as wavelength-dependent, it is recommended in this case to find out more details in the original reference.

Columns 17 through 20 are used for binary and multiple stars, giving separation, position angle, brightness ratio and type, in this order. An empty type field, or "D" or "B", refer to a double star. Other types are "T" for triple, "Q" for quadruple, "M" for multiple (which may refer to triples and quadruples too). In these cases, the parameters reported are those for two stars in the system, usually the brightest components. A code "S" was used, for those cases in which the binary nature has been disputed, and that could actually be single stars. Column 21 reports a comment, either inferred from the original paper or added by the present authors. In several cases, such comments offer some indication of the quality of the measurement.

Columns 22 and 23 list right ascension and declination, at epoch 2000.0. The format is hh.mmsss and dd.mmsss. Columns 24 and 25 list the proper motions, in arcsec/year. Values of zero indicate missing information. Coordinates and proper motions have been extracted automatically from the Simbad database in most cases.

Columns 26 through 28 list the $B, V$ and $K$ magnitudes, when available. The first two have been queried from the Simbad database. Regarding the $K$ magnitudes, we have used our own photometry, as well as measurements obtained with the DENIS instrument to complement the basic data queried from Simbad and standard catalogs. This has been described in Sect. 4.

Columns 29 through 32 are extracted from Simbad, and list respectively the spectral type, a variability flag, the parallax (in mas) and the radial velocity (in $\mathrm{km} \mathrm{s}^{-1}$ ). Columns 33 and 34 report "Y" when additional photometric and spectroscopic measurements are available from own investigations, otherwise $\mathrm{N}$ is shown. These additional 
Table 1. Sample of the catalog. Columns 1 through 42 are commented in the text.

\begin{tabular}{|c|c|c|c|c|c|c|c|c|c|c|c|c|c|}
\hline (1) & $(2)$ & \multicolumn{3}{|c|}{ (3) } & (4) & (5) & \multicolumn{2}{|l|}{$\overline{(6)}$} & \multicolumn{2}{|c|}{$\overline{\overline{(7)}}$} & \multicolumn{3}{|c|}{$\overline{~(8)}$} \\
\hline 308 & 2499 & \multicolumn{2}{|l|}{ iktau } & & Com & $\mathrm{IRC}+10050$ & \multicolumn{2}{|c|}{ IRAS $03507+1115$} & \multicolumn{2}{|c|}{$\mathrm{V}^{*} \mathrm{IK}$ Tau } & \multicolumn{3}{|c|}{ JP11 5529} \\
\hline 2146 & 2118 & \multicolumn{2}{|c|}{ gamsge } & & Diam & SAO 105500 & \multicolumn{2}{|c|}{$\mathrm{IRC}+20445$} & \multicolumn{2}{|c|}{ HD 189319} & \multicolumn{3}{|c|}{$\mathrm{AG}+191953$} \\
\hline 39 & 2688 & \multicolumn{2}{|c|}{ alfcas } & & Diam & SAO 21609 & \multicolumn{2}{|c|}{$\mathrm{IRC}+60017$} & \multicolumn{2}{|c|}{ HD 3712} & \multicolumn{3}{|c|}{$\mathrm{AG}+5665$} \\
\hline 185 & 1471 & \multicolumn{2}{|c|}{ mu.cet } & & Bin & SAO 110723 & \multicolumn{2}{|l|}{ HD17094 } & \multicolumn{2}{|c|}{$\mathrm{AG}+09255$} & \multicolumn{3}{|c|}{ IRAS $02422+0954$} \\
\hline 196 & 2464 & \multicolumn{2}{|c|}{ sao93166 } & & UR & SAO 93166 & \multicolumn{2}{|l|}{ IRC +10032} & \multicolumn{2}{|c|}{ HD 17973} & \multicolumn{3}{|c|}{$\mathrm{AG}+14254$} \\
\hline 685 & 1104 & \multicolumn{2}{|c|}{ dvtau } & & Diam & SAO 94604 & \multicolumn{2}{|l|}{ IRC+20111 } & \multicolumn{2}{|c|}{ HD 36231} & \multicolumn{3}{|c|}{$\mathrm{AG}+18453$} \\
\hline (9) & $(10)$ & $(11)$ & $(12)$ & $(13)$ & (14) & $\begin{array}{ll}(15) \quad(16) \\
\end{array}$ & $(17)$ & $(18)$ & $(19)$ & $(20)$ & & $\overline{(21)}$ & \\
\hline LBI & 11 & & & & & $\mathrm{Y}$ & & & & & period & shell & structure \\
\hline LBI & K & 4.60 & 1.10 & & 4.70 & 1.10 & & & & & & & \\
\hline LBI & 0.8 & 5.93 & 0.05 & & 5.64 & 0.05 & & & & & & & \\
\hline LO & $\mathrm{K}$ & & & & & & 7.0 & 63 & 71 & $\mathrm{Q}$ & & & \\
\hline $\mathrm{LO}$ & $\mathrm{K}$ & & & 2.00 & & & & & & & & & \\
\hline $\mathrm{LO}$ & K & 3.79 & 0.13 & & & & & & & & & & \\
\hline & $(22)$ & & $(23)$ & & $(24)$ & $(25)$ & $(26)$ & $(27)$ & $(28)$ & $\overline{(29}$ & & & $(30)$ \\
\hline & .53286 & & 1.242 & & 0.0 & 0.0 & & 11.9 & -1.24 & M6me... & & & :V4 \\
\hline 19. & 845428 & 19.2 & 3173 & & .06437 & 0.02258 & 5.11 & 3.53 & -0.29 & MOIII & & & \\
\hline & 030441 & 56.3 & 1439 & & .05036 & -0.03217 & 3.43 & 2.25 & & K0IIIa & & & \\
\hline & 456542 & 10.0 & 5093 & & .28516 & -0.0304 & 4.58 & 4.20 & 3.40 & F0IV & & & $\mathrm{V} ?$ \\
\hline & 338482 & 14.4 & 1291 & & .00199 & -0.00416 & 9.29 & 7.57 & 2.71 & M... & & & \\
\hline & 106347 & 18.3 & 3739 & & -0.0043 & -0.035 & 9.8 & 8.4 & 1.4 & M... & & & :V3 \\
\hline$(31)$ & $(32)$ & $(33)$ & $(34)$ & $(35)$ & & $(36)$ & $(37)$ & $(38)$ & $(39)$ & $(40)$ & $(41)$ & $(42)$ & \\
\hline & 46.0 & $\mathrm{~N}$ & $\mathrm{~N}$ & HDB & 1997 & & & & & & & & \\
\hline 11.9 & -32.8 & $\mathrm{~N}$ & $\mathrm{~N}$ & DBa1 & 996 & & & & 0.95 & $2.7 \%$ & 0.06 & $7.4 \%$ & \\
\hline 14.27 & -3.8 & $\mathrm{~N}$ & $\mathrm{~N}$ & MJS1 & 991 & & & & 0.918 & $0.2 \%$ & 0.063 & $1.3 \%$ & \\
\hline 38.71 & 30.4 & $\mathrm{~N}$ & $\mathrm{~N}$ & $\mathrm{RRC}$ & 2000 & & & & & & & & \\
\hline 1.57 & & $\mathrm{~N}$ & $\mathrm{~N}$ & TC20 & & & & & 0.990 & & 0.303 & & \\
\hline & & $\mathrm{N}$ & $\mathrm{N}$ & $\mathrm{RRL}$ & 998 & RFR1999 & RBC1996 & & 0.966 & $0.2 \%$ & 0.131 & $3.4 \%$ & \\
\hline
\end{tabular}

results are visible only in the full version of the catalog, in which they can be accessed as hyperlinks.

Columns 35 through 38 report a code for the original reference. A total of 154 references have been used, of which 100 and 54 for LBI and LO measurements respectively. Regarding these latter, we note that the use of the WF and DSFILE catalogs as described in Sect. 5.2 has effectively replaced all references prior to 1986. Additionally, CHARM contains a large number of hitherto unpublished LO observations available in the private archive of one of us (AR). All references are hyperlinked, including the contents of the paper, in the full version. The list of references for the ASCII version of the catalog is available together with the catalog, and is not reported here.

The last four columns are to be used as estimates of the visibility of the source, and are of interest mainly for interferometer users. In the full version of the database, these columns report embedded computations, which permit the user to change wavelength and baseline. In the fixed ASCII version, Cols. 39 and 40 report the visibility and the (relative) error, on the basis of the angular size, at the wavelength of $2.2 \mu \mathrm{m}$ on a baseline of $20 \mathrm{~m}$. This information is replicated in Cols. 41 and 42 for a baseline of $200 \mathrm{~m}$. The angular size is derived from the data in Cols. 12 through 17 when applicable (binary and multiple stars are excluded). These values are only indicative, as the source could be more complex than the simple structure of the table can accommodate, or could have a wavelength-dependent size.

\section{Results and statistics}

As mentioned in Sect. 5.3, in many cases more than one entry might be present for a given source, either because measurements were obtained at various wavelengths, or because results were presented by different authors. For this, it is helpful to use the list of cross-identifications and associations provided separately. A total of 1625 unique sources are present in CHARM.

The relative incidence of the LO and LBI techniques referenced in CHARM is approximately similar, if one considers that we have made use of the WF and DSFILE 
Table 2. List of source codes used in CHARM.

\begin{tabular}{llr}
\hline \hline Code & \multicolumn{1}{c}{ Type of source } & \# Entries \\
\hline Diam & Diameter measured & 1064 \\
Bin & Binary or mult. system & 363 \\
UR & Object unresolved & 890 \\
Com & Complex Geometry & 115 \\
\hline
\end{tabular}

Table 3. Number of publications referenced in CHARM, based on LO and LBI results.

\begin{tabular}{ccccc}
\hline \hline Year & $<1990$ & $\begin{array}{c}1990-1995 \\
\text { and 1995 }\end{array}$ & $>1995$ & Total \\
\hline LBI & 25 & 17 & 58 & 100 \\
LO & $5^{a}$ & 20 & 29 & $54^{a}$ \\
\hline
\end{tabular}

${ }^{\mathrm{a}} \mathrm{WF}$ and DSFILE used for older references, see text.

sources for older references (see Sect. 5.2). However, the time distribution of the results obtained by LO and LBI is very different. LO have produced a rather constant rate of papers, while LBI is experiencing a sharp increase in recent years, thanks also to the spread of new facilities. This is schematically summarized in Table 3.

We show in Fig. 3 the distribution of $V$ and $K$ magnitudes for the entries present in CHARM. It should be noted that this is only a first approximation, since we did not attempt to remove from this figure the bias introduced by those entries which refer to the same source. Note also that not all entries have both a $V$ and a $K$ magnitude. Nevertheless, Fig. 3 illustrates quite well the broad range of magnitudes covered by CHARM. It can be noted that both the median and the faint-end tail of the $V$ magnitude distribution extend to fainter magnitudes, than those of the $K$ magnitude distribution. This is easily explained, not only because statistically the sources have $V-K>0$ as is expected for a wide sample of stellar sources, but also because the best targets for high angular resolution methods are often cool and obscured stars, with very red colors. It can be appreciated how the magnitude distribution is sufficiently wide, to provide sources for science verification and calibration also of large facilities.

It is also interesting to examine the distribution of measured angular diameters available in CHARM. For this, we have plotted in Fig. 4 an histogram of angular diameters, where one should note the following approximations: we have mixed uniform disk and limb-darkened diameters, using the latter when the former was not available; additionally, no distinction has been made regarding the wavelength of observation; finally, also in this case we have made no distinction for those sources which may be represented several times under different entries. Therefore the histogram of Fig. 4 is biased, but given the large number of measurements present it is nevertheless a sufficient approximation. The distribution peaks at about 5 mas: this represents the optimal range of resolution for both LO and LBI, and results from the combination of the

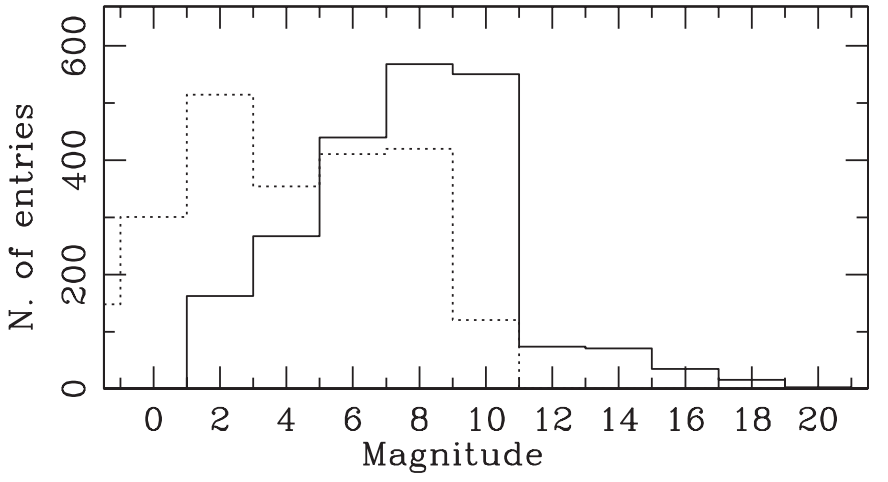

Fig. 3. Distribution of $V$ and $K$ magnitudes (solid and dashed line respectively).

typical angular resolution of these techniques on one side, with the typical size of the sources which can be accessed given the typical limiting magnitudes. While sources with an angular size of 5 mas or more are probably inadequate for the needs of a large modern interferometer (see also Table 4 below), it can be noted from Fig. 4 that a significant number of sources with diameters smaller than this are also available in CHARM. Regarding those sources at the large diameter end of the distribution, a few extreme cases are in fact due to measurements obtained at long wavelengths on peculiar sources: in fact, they represent more the size of circumstellar emission rather than a photospheric diameter. A few entries are present in CHARM, with an angular size above 200 mas, which also fall in the category just mentioned and are not represented in Fig. 4.

Finally, we note that we did not include in Fig. 4 sources with an upper limit on the diameter. A total of 45 are included in CHARM, with an average upper limit of 3.1 mas. Given the small size of the sample and the overlap with the range of diameters for the resolved sources, this contribution would not alter significantly the statistics shown in Fig. 4.

We determined similar statistics also for the separation of the binary systems referenced in CHARM, and we show the result in Fig. 5. As for the case of the angular diameters, we have mixed observations of a same source listed under different entries. Additionally, we note that in fact in all measurements obtained by LO (the majority of binary stars entries listed in CHARM), the values refer to projected separations only. However, we do not expect this to introduce a significant bias in Fig. 5, since the average factor for a random orientation of the true position angle should be only $2 / \pi$. Note however that in the case of multiple systems ( $13 \%$ of the total of binary systems included in CHARM), we have used only the separation of the two brightest components in the system. Although the statistics of binary systems is smaller than those of the angular diameters and the typical separation is $\approx 10$ times larger than the peak of the distribution in Fig. 4, it can be noted that a significative numbers of binary systems could also be used in principle for the calibration and verification of large modern interferometers. One should of 


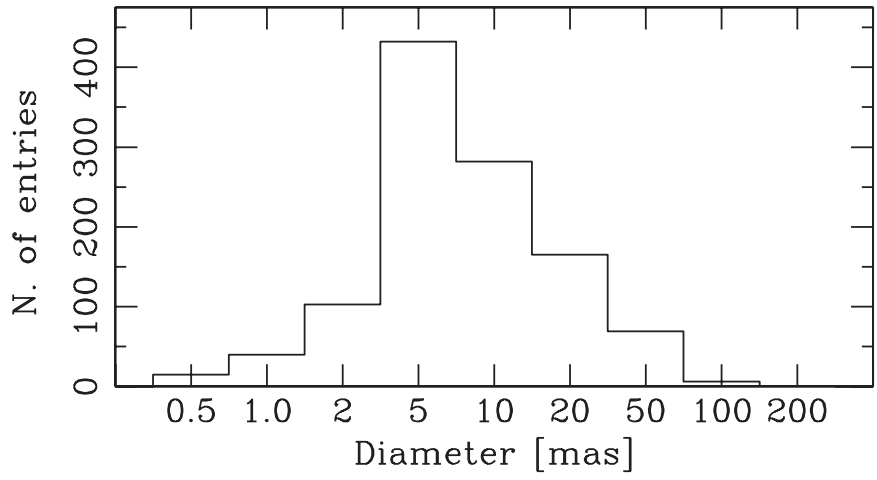

Fig. 4. Distribution of diameters. Note that the scale of the horizontal axis is arbitrary.

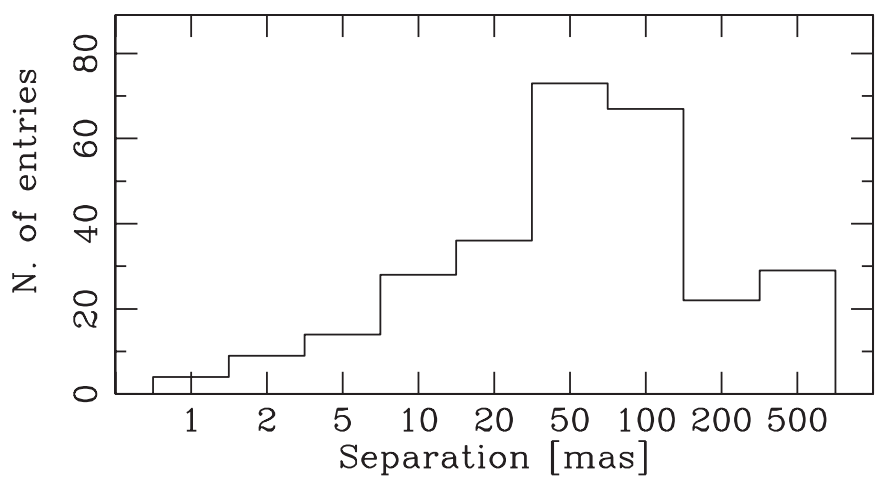

Fig. 5. Distribution of binary separations.

course take into account also the brightness ratio of the components in order to assess the feasibility of a measurement, but on the other hand it should be remarked that a binary system with well-known properties is well-suited to characterize an interferometer, since its visibility does not suffer from the sharp decrease beyond the first minimum, as is the case for angular diameters.

In Figs. 6-7 we show the distribution of the CHARM entries in terms of spectral type and luminosity class. As mentioned before, these distributions are biased by the fact that we treat all entries as independent. However, this effect is not expected to be dominant, given the relatively large statistics. We note at the same time that not all entries have an explicit or unique spectral type and luminosity class. We have restricted the distributions in Figs. 6-7 to those entries which were explicitly classified. These included 2155 and 1378 entries for the spectral type and luminosity respectively ( $89 \%$ and $57 \%$ of the CHARM sample respectively). As can be seen, the LO and LBI techniques have been used mainly to measure late-type giants.

One of the main goals of a compilation such as CHARM is to provide a list of sources which can be used for the purposes of calibration and science verification of modern large interferometers. For this, it is not only necessary to provide a list of measured angular diameters, but it is essential to associate an error to them. In this way, meaningful estimates of the expected visibility for a given baseline and wavelength can be made, along with

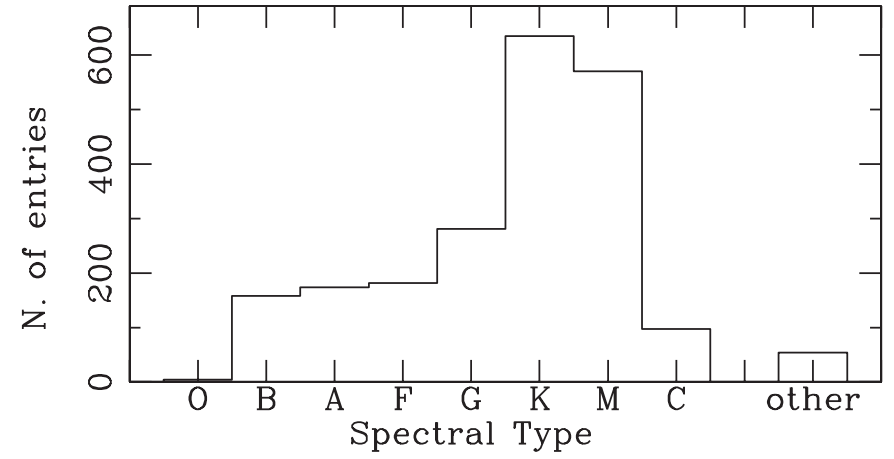

Fig. 6. Distribution of spectral type.

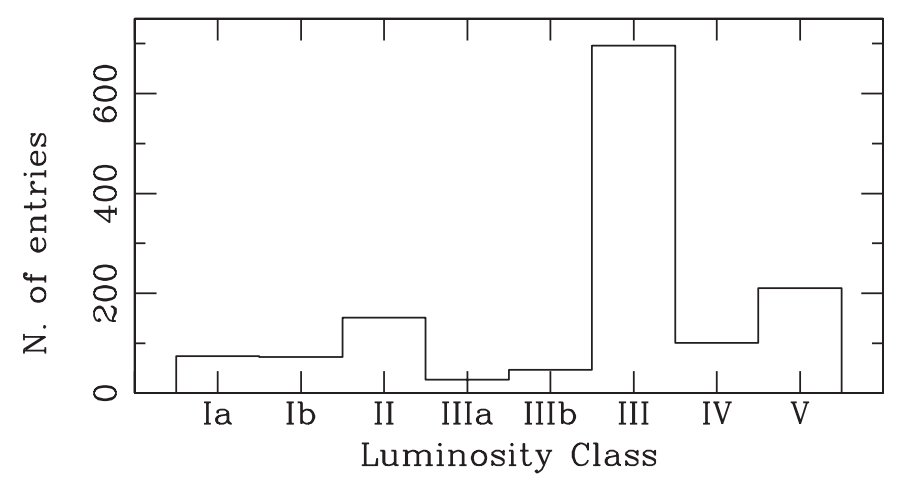

Fig. 7. Distribution of luminosity class.

the error. In CHARM, a large majority of the sources with a measured angular diameter have also an associated error (91\% of the cases). In order to evaluate the practical utility of CHARM for calibration and science verification purposes, we have made some statistics on the expected visibilities for a few wavelengths and baselines. For this, we have used all the entries with a measured diameter and error, using either the uniform disk or the limb-darkened value, depending on availability and without corrections. Note also that we have used all entries as independent although, as stated earlier, different entries can refer in practice to the same source. We have also neglected to associate a diameter measured in a given wavelength with the visibility at the same wavelength, mixing in fact all measurements together. Note finally that no threshold for the limiting magnitude has been applied.

This analysis is shown in Table 4 , where it can be seen that at least for the near-IR and thermal-IR ranges there is always a good number of sources available, with a visibility in a favorable range (we have assumed a range of 20-80\%), while at visual wavelengths CHARM has a limited availability for baselines longer than $100 \mathrm{~m}$. However, when one additionally requires a minimum accuracy in the visibility, the situation changes considerably. With a visibility accuracy of $1-2 \%$, the availability of sources in CHARM for the near-IR and thermal-IR is still sufficient, but below $0.5 \%$ the numbers are considerably reduced. The situation is naturally much worse at visual wavelengths, were no sources with a visibility accuracy better than $2 \%$ are available in CHARM for baselines of $100 \mathrm{~m}$ and longer. 
Table 4. Summary of entries available in CHARM, within an optimal range of visibility and for three levels of relative visibility accuracy.

\begin{tabular}{|c|c|c|c|c|c|}
\hline $\begin{array}{c}(1) \\
\lambda\end{array}$ & $\begin{array}{c}(2) \\
\text { Basel. }\end{array}$ & $\begin{array}{c}(3) \\
0.2 \leq V \leq 0.8\end{array}$ & (4) & $\begin{array}{c}(5) \\
\Delta V / V\end{array}$ & (6) \\
\hline$(\mu \mathrm{m})$ & $(\mathrm{m})$ & & $\leq 0.5 \%$ & $\leq 1 \%$ & $\leq 2 \%$ \\
\hline \multirow{3}{*}{0.8} & 50 & 287 & 3 & 9 & 17 \\
\hline & 100 & 81 & 1 & 1 & 4 \\
\hline & 200 & 36 & - & - & - \\
\hline \multirow{3}{*}{2.2} & 50 & 354 & 7 & 22 & 43 \\
\hline & 100 & 379 & 6 & 15 & 55 \\
\hline & 200 & 142 & - & 2 & 6 \\
\hline \multirow{3}{*}{10} & 50 & 88 & 13 & 32 & 46 \\
\hline & 100 & 226 & 4 & 10 & 49 \\
\hline & 200 & 337 & 8 & 18 & 37 \\
\hline
\end{tabular}

In summary, Table 4 shows quantitatively the wellknown situation, that modern interferometers with baselines above $100 \mathrm{~m}$ and with potential accuracies below $1 \%$, will need to establish their own network of calibrators. This is an effort that will strongly benefit from collaborations between the various facilities. Such a network of calibrators will need to rely on a boot-strapping approach using shorter baselines and known diameters. Additionally, it will be essential to use the best available indirect estimates, and further extensions of the presently available lists (Cohen et al. 1999; Blackwell \& Lynas-Gray 1994) are highly desirable.

CHARM can also be used as a source of informations for other purposes than those mentioned above. For example, we have tried to assess the reliability of independent measurements obtained by LO and LBI. For this, we have plotted in Fig. 8 an histogram of the results obtained at different wavelengths by the two techniques on a wellknown star with a stable diameter. Within the limits of the available statistics, it can be seen that the two techniques yield essentially the same average, although LBI results have $\approx 1 / 2$ of the spread of the LO ones. It must however be stressed that Aldebaran is a rather difficult object for LO: paradoxically, it is too large and the diffraction fringes due to the lunar limb are almost completely cancelled. Note also that for this histogram we have made no distinction on the wavelength of observation.

Figure 9 shows another application, for which we have selected all available measurements relative to 3 stars, 2 of which are known to be variable ( $\mathrm{R}$ Leo and $\mathrm{Tx}$ Psc), and one which is presumed to be stable (Aldebaran). The CHARM entries reflect very well the different situations for the various stars, with $\mathrm{R}$ Leo showing additionally a marked dependence of the variation amplitude on the wavelength. It should be stressed that the horizontal axis in Fig. 9 is not related with time: the entries are in the same order as in CHARM.

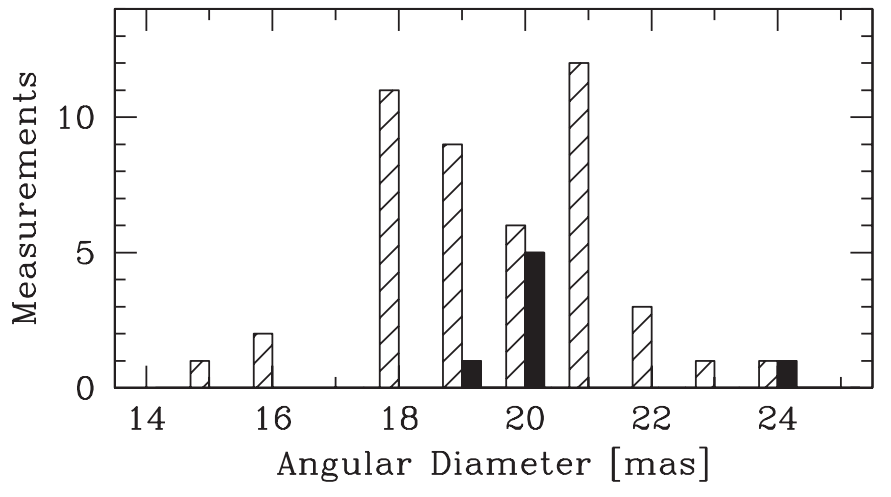

Fig. 8. Distribution of the angular diameter measurements of Aldebaran ( $\alpha$ Tau), as obtained by LO and LBI (dashed and solid histograms, respectively). See discussion in the text.

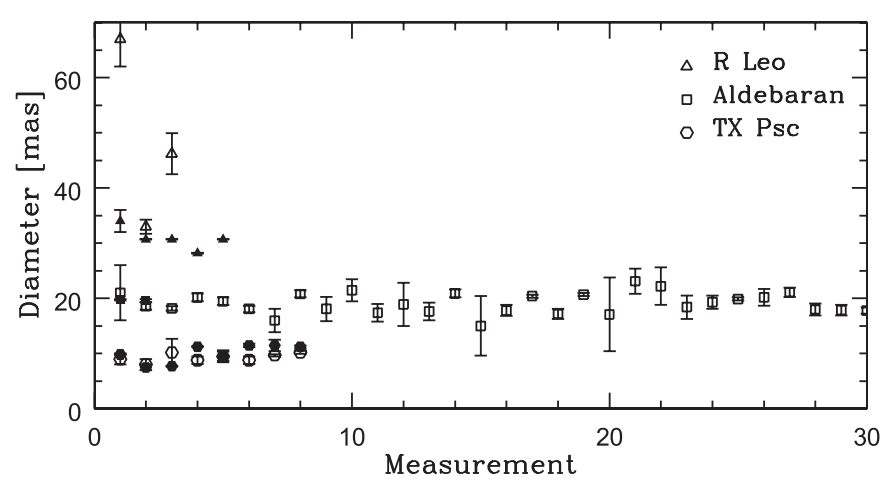

Fig. 9. Distribution of angular diameter measurements for 3 objects Aldebaran ( $\alpha$ Tau), R Leo, TX Psc as obtained in the visible and in the $K$ band (empty and solid symbols respectively). See discussion in the text.

\section{Conclusions}

We have collected all measurements obtained by the techniques of lunar occultations and long-baseline interferometry at visible and infrared wavelengths that are available in the literature, from the WEB and from private sources, concerning high angular resolution measurements by the techniques of lunar occultations and long baseline interferometry. Results such as angular diameters, binary stars, circumstellar shells are included. We have included, whenever possible, also determinations of unresolved sources. Our compilation was carried out until mid2001, and should be considered complete at least throughout the year 2000 .

The result is a database of 2432 measurements of 1625 sources. It includes photometry extracted either from available catalogs or obtained specifically for this work, as well as a collection of informations regarding spectral types, variability, cross-identifications, and more. This database, dubbed CHARM, includes also a complete set of the original papers. The information is presented as an ASCII table, and provided in a full version on CD-Rom upon request. This latter includes information organized in a system of directory structures, and is based on an 
Excel file that provides functionalities for sorting, filtering, embedded computations and hyperlinks.

We have provided a brief overview of the principles of the two techniques under consideration, including a discussion of their main limitations. We have presented and discussed some basic statistical inferences that can be made with CHARM, such as the distribution of magnitudes, angular diameters, binary separations, and the possible utility of this database to select sources suitable for calibration and verification of large modern interferometers.

The material presented in this work should be sufficient for the needs of interferometers of less than $100 \mathrm{~m}$ operating in the near-IR with accuracies around the $1 \%$ level. However, in the case of longer baselines or shorter wavelengths, and especially for high accuracy observations, it will be mandatory to establish a new set of interferometric calibrators.

Acknowledgements. This research has made use of the Simbad database, operated at the Centre de Données Astronomiques de Strasbourg (CDS), and of NASA's Astrophysics Data System Bibliographic Services (ADS).

\section{References}

Baldwin, J. E., Beckett, M. G., Boysen, R. C., et al. 1996, A\&A, 306, L13

Blackwell, D. E., \& Lynas-Gray, A. E. 1994, A\&A, 282, 899

Cohen, M., Walker, R. G., Carter, B., et al. 1999, AJ, 117, 1864

Colavita, M. M., \& Wizinowich, P. L. 2000, SPIE Proc. 4006, Interferometry in Optical Astronomy, ed. P. Léna, \& A. Quirrenbach, 310

Delplancke, F., Lévêque, S., Kervella, P., Glindemann, A., \& D' Arcio, L. 2000, SPIE Proc. 4006, Interferometry in Optical Astronomy, ed. P. Léna, \& A. Quirrenbach, 365
Egan, M. P., Price, S. D., Moshir, M. M., et al. 1999, The Midcourse Space Experiment Point Source Catalog, Version 1.2, Air Force Research Lab. Technical Rep. AFRLVS-TR-1999-1522

Esposito, S., Riccardi, A., \& Femenìa, B. 2000, A\&A, 353, L29

Evans, D. S., McWilliam, A., Sandmann, W. H., \& Frueh, M. 1986, AJ, 92, 1210

Fracassini, M., Pasinetti, L. E., \& Manzolini, F. 1981, A\&ASS, 45,145

Gezari, D. Y., Pitts, P. S., \& Schmitz, M. 1999, Catalog of Infrared Observations, 5

Glindemann, A., Coudé du Foresto, V., Delplancke, F., et al. 2000, SPIE Proc. 4006, Interferometry in Optical Astronomy, ed. P. Léna, \& A. Quirrenbach, 2

Hanbury Brown, R., Davis, J., \& Allen, L. R. 1974, MNRAS, 167,121

Hartkopf, W. I., McAlister, H. A., \& Mason, B. D. 2001, AJ, 122,3480

Kimeswenger, S., Lederle, C., Armsdorfer, B., et al. 2002, $\mathrm{A} \& \mathrm{~A}$, in preparation

Labeyrie, A. 1975, ApJ, 196, L71

Michelson, A. A., \& Pease, F. G. 1921, ApJ, 53, 249

Nather, R. E., \& McCants, M. M. 1970, AJ, 75, 963

Neugebauer, G., \& Leighton, R. B. 1969, The Two-Micron Sky Survey, NASA SP-3047

Pasinetti Fracassini, L. E., Pastori, L., Covino, S., \& Pozzi, A. 2001, A\&A, 367, 511

Richichi, A. 1989, A\&A, 226, 366

Richichi, A., Lisi, F., \& Di Giacomo, A. 1992, A\&A, 254, 149

Richichi, A., Fabbroni, L., Ragland, S., \& Scholz, M. 1999, A\&A, 344, 511

Richichi, A, Calamai, G, \& Stecklum, B. 2002, A\&A, 382, 178

Ridgway, S. T. 1977, AJ, 82, 511

Ridgway, S. T., \& Roddier, F. 2000, SPIE Proc. 4006, Interferometry in Optical Astronomy, ed. P. Léna, \& A. Quirrenbach, 940

Skrutskie, M. F., Schneider, S. E., Stiening, R., et al. 1997, The Two Micron All Sky Survey, Proc. Workshop The Impact of Large Scale Near-IR Sky Surveys, 25

White, N. M., \& Feierman, B. H. (WF) 1987, AJ, 94, 751 